\title{
The modulation of sirtuins and apoptotic proteins in rats after exhaustive exercise
}

\author{
Gabriella Marfe $^{1 *}$, Vincenzo Manzi ${ }^{2}$, Marco Tafani ${ }^{3}$, Bruna Pucci ${ }^{4}$, Alessandra Gambacurta ${ }^{1}$, \\ Matteo Antonio Russo ${ }^{3,4}$, Paola Sinibaldi-Salimei ${ }^{1}$
}

${ }^{1}$ Department of Experimental Medicine and Chirurgy, University of Rome "Tor Vergata", Rome, Italy

${ }^{2}$ School of Sport and Exercise Sciences, University "San Raffaele", Rome, Italy

${ }^{3}$ Department of Experimental Medicine, La Sapienza University, Rome, Italy

${ }^{4}$ Department of Cellular and Molecular Pathology, IRCCS San Raffaele Pisana, Rome, Italy

Email: "gabriellamarfe@libero.it

Received 18 June 2012; revised 28 July 2012; accepted 9 August 2012

\begin{abstract}
A large body of evidence shows that a single bout of strenuous exercise induces oxidative stress in circulating human lymphocytes leading to lipid peroxidation, DNA damage, mitochondrial perturbations, and protein oxidation. In a training experiment, Wistar rats were divided into control group (CG) and exercise group (EG). After a running level exercise until exhaustion, we observed an increase in the mRNA content and protein expression of SIRT1 and SIRT7 in the EG compared to the CG. Moreover, such training exercise did not change mRNA transcripts and protein expression of FOXO3A and GADD45. We also observed an increase of pro-apoptotic protein bax and a decrease of the anti-apoptotic protein bcl-2 in the EG. Accordingly, we observed a caspase-3 activation and poly (ADP-ribose) polymerase (PARP) cleavage only in EG rats. Statistical analysis of the data showed a significant correlation between SIRT1 and SIRT7 expression and apoptotic proteins such as bax, bcl-2 in both tissues. We conclude that, in both muscle, such exercise activates both a damaging apoptotic mechanism with bax increase and bcl-2 decrease and a counterbalancing protective mechanism with SIRT1 and SIRT7 increase.
\end{abstract}

Keywords: Cardiac Muscle; Skeletal Muscle; Bax; Bcl-2; SIRT1; SIRT7

\section{INTRODUCTION}

It is widely accepted that exhaustive exercise promotes drastic homeostatic changes in our internal milieu, constituting a challenge to the cells' ability to survive under

\footnotetext{
${ }^{*}$ Corresponding author.
}

this stressful situation [1]. The enhanced metabolic and mechanical demands imposed by exercise to several organs and tissues, particularly to skeletal and cardiac muscles may overwhelm their cellular capacity to maintain or restore homeostasis, hence enhancing the expression of several markers of cell damage/death [2] tissue inflammation and immunological alterations in the blood [3]. In fact, exercise-associated elevations of skeletal muscle and cardiac biomarkers can be detected in both elite and recreational athletes, especially after prolonged and strenuous exercises [4]. At a tissue level, the cellular fate to die or to survive to this stressful situation depends from the magnitude of the stimulus and from the cellular ability to repair damages. The latter aspect being ultimately determined by changes occurred in gene expression, particularly of those genes interfering with the control of metabolism $[5,6]$ apoptosis $[7,8]$. Indeed, in heart and skeletal muscle it is well-known that a single bout of exercise may promote huge alterations in the expression of nuclear and mitochondrial genes as an attempt to neutralize the harmful effects of this metabolic stress [9]. Although many cells may undergo necrosis, the adopted strategy for damaged cells might pass through a genetically controlled suicide, which depends of the gene expression ratio of proteins that initiate (e.g., bax, Fas, p.53, etc.) or inhibit (e.g., bcl-2, bcl-XL) apoptosis [10]. Despite not yet well-documented in cardiac muscle [2], the occurrence of apoptosis induced by exercise has been already described in skeletal muscle fibers [11].

It is believed that intracellular alterations in $\mathrm{Ca}^{2+}$ and in the energy status are main cellular signals leading to the altered gene response induced by exercise, through the activation of several downstream kinases, such as the AMP kinase and $\mathrm{Ca}^{2+}$-calmodulin-activated kinases [2]. These kinases will further activate several transcription factors. Among those, PGC- $1 \alpha$ acts as a transcriptional 
co activator for different metabolic genes being currently considered a key controller of skeletal muscle adaptations induced by exercise [12]. However, in an elegant study performed in skeletal muscle of knockout mice [13] the authors clearly showed that, apart PGC-1alpha, other factors are as well decisive to mediate this gene response induced by acute exercise. Indeed, changes in energy homeostasis could be also detected by other specific and sensing molecules, which in parallel with Kinases and PGC1alpha may play an important role in the modulation of gene expression [14]. Sirtuins, a recently identified mammalian family of regulatory molecules (SIRT1 to SIRT7), are strong candidates to play this role during exercise since they have been implicated in the control of critical cellular processes such as differentiation, proliferation, apoptosis, and metabolism [15]. The alteration of cytosolic $\mathrm{NAD}^{+} / \mathrm{NADH}$ ratio during contraction in both skeletal and cardiac muscles constitutes a potential stimulus to activate sirtuins, particularly SIRT1 [16]. SIRT1 is involved in many physiological functions, including control of gene expression, cell cycle regulation, apoptosis, autophagy, DNA repair, metabolism, and oxidative stress response. SIRT1 also deacetylates non-histone proteins such as p53, Forkhead transcription factors (FOXO) [17] and nuclear factor nKB [18]. FOXO3A has been shown to modulate various genes that control repair of damaged DNA (GADD45), reactive oxygen species (ROS) detoxification, cell cycle arrest and apoptosis. Indeed, it was recently showed that endurance exercise increases the skeletal muscle SIRT1 protein content [19]. Moreover, it is known that many of the above-referred Sirtuins targeted proteins, namely the peroxisome proliferator-activated receptor, the PGC-1alpha and the FOXO have an enhanced expression/activation during and after acute exercise [20]. Also SIRT7 constitutes a strong candidate to modulate this cellular response to acute exercise, at least in heart. In fact, SIRT7-deficient primary cardiomyocytes showed an increase in basal apoptosis and a significantly diminished resistance to oxidative and genotoxic stress [21].

In order to bring new insights to the hypothetical upregulation and contribution of Sirtuins in the control of cellular strategies associated with programmed cell death vs. survival during exercise, the purposes of the present study were: 1) To examine both in cardiac and skeletal muscle of rats the effects of a prolonged and exhaustive exercise on gene expression and protein content of SIRT1 and SIRT7; 2) To associate the magnitude of this response with the expression of several genes and proteins closely linked with the cellular response to stress, involving proapoptotic (bax, cleaved caspase-3) and anti-apoptotic proteins (bcl-2) as well as transcriptional factors controlling the cellular repair of damaged DNA (GADD45, cleaved PARP, FOXO3A).

\section{MATERIAL AND METHODS}

\subsection{Ethical Approval Animals}

Ten male Wistar rats (Charles Rivers Laboratories, Barcelona, Spain) were kept individually in appropriated cages under standard laboratory conditions $(12 / 12 \mathrm{~h}$ light/darkness, $22^{\circ} \mathrm{C} \pm 1{ }^{\circ} \mathrm{C}$ room temperature, $50 \%$ - $60 \%$ humidity) for at least 1 week (quarantine) before starting the experiments. During this period animals were allowed access to tap water and rat chow ad libitum. Housing and experimental treatment of animals were in accordance with the Guide for the Care and Use of Laboratory Animals from the Institute for Laboratory Animal Research (ILAR 1996). The local Ethics Committee approved the study and the experiments were complied with the current national laws.

\subsection{Experimental Design}

After the quarantine period, rats (weighting $338.1 \pm 4.4 \mathrm{~g}$, aged 12 - 13 weeks) were randomly divided into 2 different groups: the control group $(\mathrm{CG}, \mathrm{n}=5)$ and the exercise group $(E G, n=5)$. Animal from $C G$ were placed on a motor-drive treadmill at a constant speed of $0 \mathrm{~m} \cdot \mathrm{min}^{-1}$, and then sacrificed While, animals from EG performed a running level exercise until exhaustion on a motor-drive treadmill at a constant speed of $25 \mathrm{~m} \cdot \mathrm{min}^{-1}$, being sacrificed immediately after. The animals were initially familiarized with the treadmill at a slow velocity $\left(10 \mathrm{~m} \cdot \mathrm{min}^{-1}\right)$, being the running speed progressively increased to reach (within 5 minutes) the treadmill final speed of $25 \mathrm{~m} \cdot \mathrm{min}^{-1}$ (the exercise protocol was conducted in the laboratory of Prof. José Alberto Duarte at Research Centre in Physical Activity, Health and Leisure, Faculty of Sport, University of Porto, Porto, Portugal). Exhaustion was identified by the animals' inability to maintain the running speed even with tactile stimulation of their tails.

\subsection{Tissue Collection}

Rats were anesthetized with sodium thiopental $(60 \mathrm{mg} / \mathrm{kg}$, i.p.) and placed in a supine position. After a quick opening chest, the whole heart was rapidly excised, rinsed with ice-cold saline, carefully dried. Then, the atria and the great vessels were removed and the ventricles stored at $-80^{\circ} \mathrm{C}$ for posterior biochemical analysis. From each animal, both intact gastrocnemius muscles were also collected for further biochemical assays. Then, the tissues were minced and homogenized with an Ultra-Turrax homogenizer.

\subsection{RNA Isolation and Northern Blot}

Total RNAs from samples were isolated using Tri Reagent. Aliquots of RNA were electrophoresed on 1\% agaroseformaldehyde gels and subsequently blotted onto nylon membranes. The membrane was then UV cross-linked, 
and hybridized to ${ }^{32} \mathrm{P}$-labelled probe. The relative amount of transcript was quantified using a Gel-Doc phosphorimager and Quantity One software and normalized by the intensity of $\beta$-actin.

\subsection{Protein Extraction and Western Blot Analysis}

Tissues were homogenized directly into lysis buffer. The lysates were centrifugated at $14,000 \times \mathrm{rpm}$ for $10 \mathrm{~min}$. Protein concentrations were determined by the Bradford assay. Equivalent amounts of proteins were loaded and electrophoresed on SDS-polyacrylamide gels. Subsequently, proteins were transferred to nitrocellulose membranes. After blocking with Tris-buffered saline-BSA, the membrane was incubated with the following primary antibodies: anti-caspase-3, anti-PARP, anti-bax, anti-bcl-2, antiSIRT1, anti-SIRT7, anti-FOXO3A, anti-GADD45 and anti-b-actin. Membranes were then incubated with the appropriate horseradish peroxidase-conjugated secondary antibody, and the reaction was detected with an enhanced chemiluminescence system. The relative amount of protein expression was quantified using a Gel-Doc phosphorimager and Quantity One software and normalized by the intensity of $\beta$-actin.

\subsection{Statistical Analysis}

The results are expressed as means $\pm \mathrm{SD}$. A one-way between-groups multivariate analysis of variance was performed to investigate any significant differences in physiological data between groups. Preliminary assumption testing was conducted to check for normality, linearity, univariate and multivariate outliers, homogeneity of variance-covariance matrices, and multicollinearity. Significance was set at $p \leq 0.05$. The SPSS statistical software package (version 13.0.1 for Windows; SPSS, Inc., Chicago, IL) was used for all statistical analyses.

\section{RESULTS}

\subsection{Exercise Increases Expression Levels of SIRT1 and SIRT7}

Immediately following a bout of prolonged and exhaustive exercise, the mRNA levels of SIRT1 and SIRT7 were increased in both cardiac and skeletal muscle tissues of EG rats compared to CG rats (Figures 1(a) and (c)). In particular, we found a statistically significant difference in SIRT1 mRNA levels in both cardiac and skeletal muscle tissues between two groups $(\mathrm{p}=0.0004 ; \mathrm{p}=0.0001)$.

To obtain further evidence regarding the role of SIRT1 and SIRT7 in this kind of exercise, we performed Western Blot analysis immediately after a bout of run. As shown in Figures 1(b) and (d), the expression of these proteins were increased in both tissue, while we did not observe any difference of expression of these proteins in CG. Statistical analysis of the data revealed that SIRT1 protein expression in cardiac muscle as well as SIRT1 and SIRT7 protein expression in skeletal muscle were not statistically different between two groups $(p=0.10 ; p=$ $0.44 ; p=0.79$ ). By contrast, the differences of SIRT7 protein expression in cardiac muscle between EC and CG were statistically different $(p=0.001)$. This finding could provide evidence that the initial adaptive increase in cardiac and skeletal muscle tissue biogenesis is mediated by the exercise-induced increase in SIRT1 and SIRT7 protein expression.

Furthermore, such data are confirmed by the mRNA transcripts and the expression levels of Lactate Dehydrogenase (LDH) as shown in Figure 1S. In fact, we observed that a prolonged and exhaustive exercise significantly increased mRNA levels and expression protein levels of $\mathrm{LDH}$ in both cardiac and skeletal muscle tissues in EG $(p=0.001 ; p=0.001$, Figures 1S(a) and (b)). Furthermore, we found that LDH transcripts and protein expression levels were statistically significant in both cardiac and skeletal muscle tissues between two groups, as shown in Figures 2S(a) and (b) $(\mathrm{p}=0.001 ; \mathrm{p}=0.001)$.

\subsection{Exercise Does Not Increase Expression Level of FOXO3A and GADD45}

To further evaluate the possibility that increased expression of SIRT1 mediates the phase of exercise-induced cardiac and skeletal muscle tissue biogenesis, we measured FOXO3A expression. We observed a decrease of FOXO3A transcripts in the cardiac and skeletal muscle tissue after a bout of prolonged and exhaustive exercise (Figure 2(a)), but we did not find a statically significant difference in both tissues between the two groups. Whereas, this exercise did not increase FOXO3A protein expression level in both tissues of EG rats compared to CG rats (Figure 2(b)). Furthermore, this exercise training influenced only mRNA transcripts but no protein expression of GADD45 in cardiac and skeletal muscle tissue (Figures 2(c) and (d)).

\subsection{Exercise Modulates the Expression Level of Pro- and Anti-Apoptotic Proteins}

Cleaved caspase-3 and PARP are key mediators of apoptosis and are measured as important markers of in vivo apoptosis. Proteolytic cleavage of caspase-3 and its substrate PARP, a hallmark of apoptosis, were increased in both tissues after exhaustive exercise. As shown in Figure 3, proteolytic cleavage of caspase-3 and PARP in cardiac and skeletal muscle samples was observed in EG but not in CG rats. In addition, Figure 4(a) shows that, in the EG rats, bax mRNA level increased in the two tissues examined $(p=0.03 ; p=0.0002)$ compared with the $C G$ 

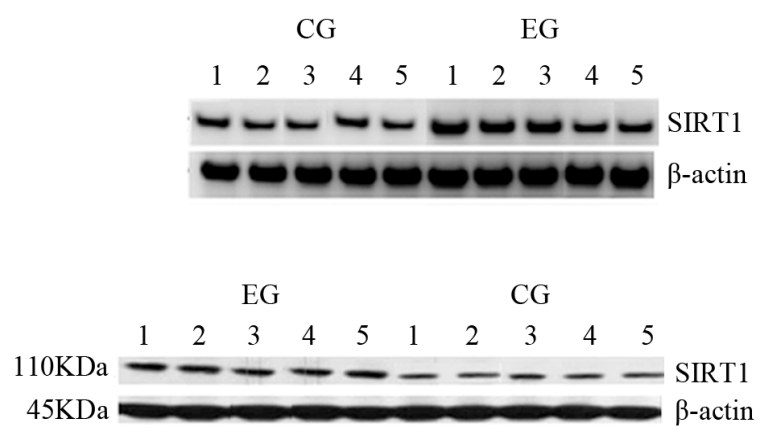

(a)

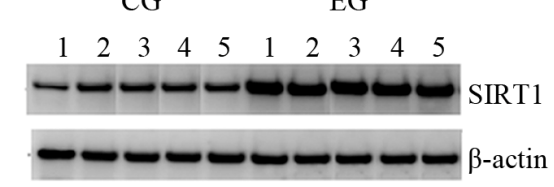

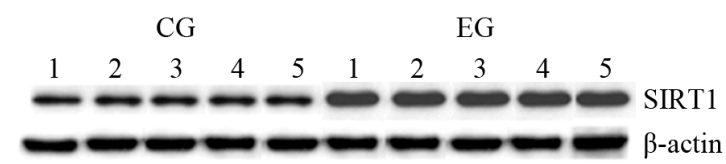

(b)
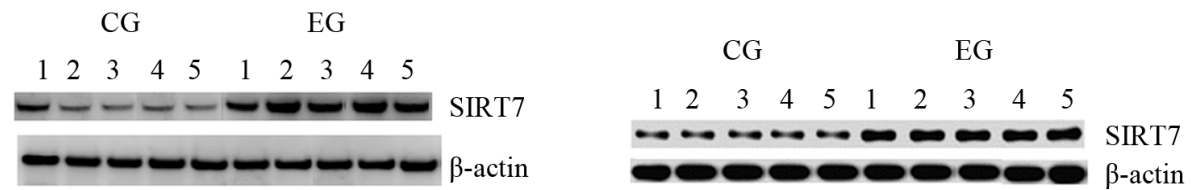

(c)

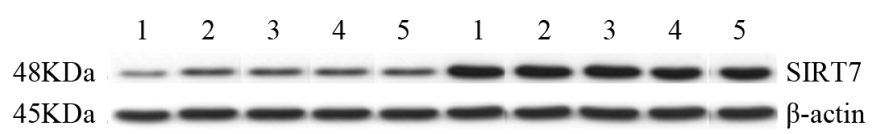

cardiac muscle tissue

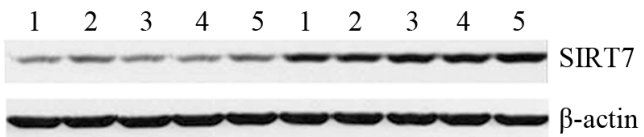

skeletal muscle tissue

(d)

Figure 1. Effects of run to exhaustion in rat cardiac and skeletal muscle tissues on the SIRT1 and SIRT7 mRNA and protein expression levels. Northern blot and Western blot analysis were performed. $\beta$-actin was used as an internal loading control. (a) Total mRNA was extracted from tissues of CG and EG rats and analyzed Northern blot. The membrane was hybridized with a ${ }^{32} \mathrm{P}$ labeled probe for SIRT1; (b) Whole cell lysates were prepared from tissues of CG and EG rats and analyzed by Western blot using SIRT1 specific antibodies. $\beta$-actin was used as a control for protein loading. The molecular weight (in $\mathrm{KDa}$ ) of protein size standards is shown on the left hand side; (c) Total mRNA was extracted from tissues of CG nd EG rats and analyzed Northern blot. The membrane was hybridized with a ${ }^{32} \mathrm{P}$ labeled probe for SIRT7; (d) Whole cell lysates were prepared from tissues of CG and EG rats and analyzed by Western blot using SIRT1 specific antibodies. $\beta$-actin was used as a control for protein loading. The molecular weight (in KDa) of protein size standards is shown on the left hand side.

rats. Similarly, Figure 4(b) shows that, bax protein expression increased in cardiac and skeletal muscle tissue in EG rats compared to $\mathrm{CG}$ rats. However, by comparing the $\mathrm{CG}$ and $\mathrm{EG}$ rats, we found only the increase in the cardiac muscle tissues of the EG rats to be statistically significant $(\mathrm{p}=0.02 ; \mathrm{p}=0.45)$. We next determined the expression levels of the anti-apoptotic protein bcl-2. Figure 4(c) shows that bcl-2 mRNA decreased in both tissues in a statically significant manner in EG rats compared to $\mathrm{CG}$ rats $(\mathrm{p}=0.0001 ; \mathrm{p}=0.001)$. By contrast, Figure 4(d) shows that the decrease in bcl-2 protein expression, observed in the EG rats, was not statically significant in cardiac and skeletal muscle tissues $(p=0.14 ; p=0.760)$ between the CG and EG rats. Furthermore, since exhaustive exercise tended to increase bax and decrease bcl-2 both at transcripts and protein expression levels, we calculated the bax:bcl-2 ratio at both levels. We found that the bax:bcl-2 mRNA ratio is statically significant in cardiac and skeletal muscle tissue between two groups $(\mathrm{p}=0.03$; $\mathrm{p}=0.002$, Figure 5(a)). By contrast, bax:bcl-2 protein ratio is statically significant only in cardiac muscle tissues between two groups ( $\mathrm{p}=0.02 ; \mathrm{p}=0.196$, Figure 5(b)).

\section{DISCUSSION}

The purpose of this study was to examine the effects of prolonged and exhaustive stress, using an exercise model, on SIRT1 and SIRT7 expression as well as on the expression of apoptotic and anti-apoptotic proteins in cardiac and skeletal muscle tissues. One of the main findings was that exhaustive exercise was associated with an increase expression of SIRT1 and SIRT7 proteins. A second important finding was that in response to prolonged and exhaustive stress, cardiac and skeletal muscle tissues showed an increased expression of the pro-apoptotic protein bax, followed by cleavage of caspase- 3 and PARP in EG. SIRT1 deacetylase and PARP respond to environmental cues, and both require NAD+ cofactor for their enzymatic activities. Recent studies have shown that PARP activation in cardiac myocytes during heart failure results in myocyte cell death by decreasing the cellular NAD levels, consequently reducing the activity of SIRT1 [22]. We have shown here that SIRT1 mRNA and protein expression increased acutely following prolonged and exhaustive exercise in both cardiac and skeletal muscle tissues. Our results suggest that 

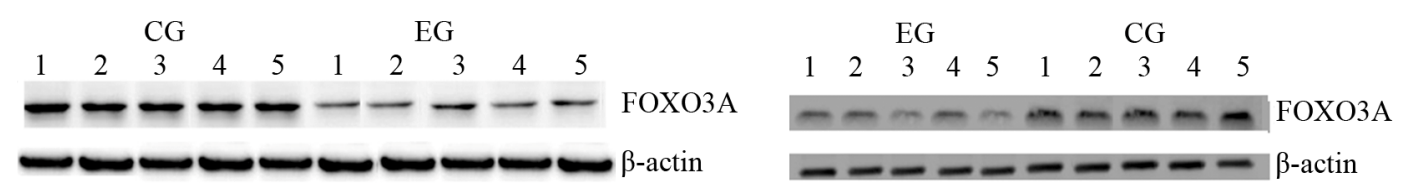

(a)

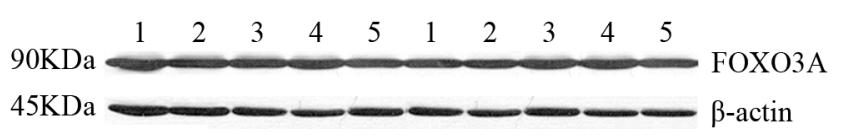

(b)
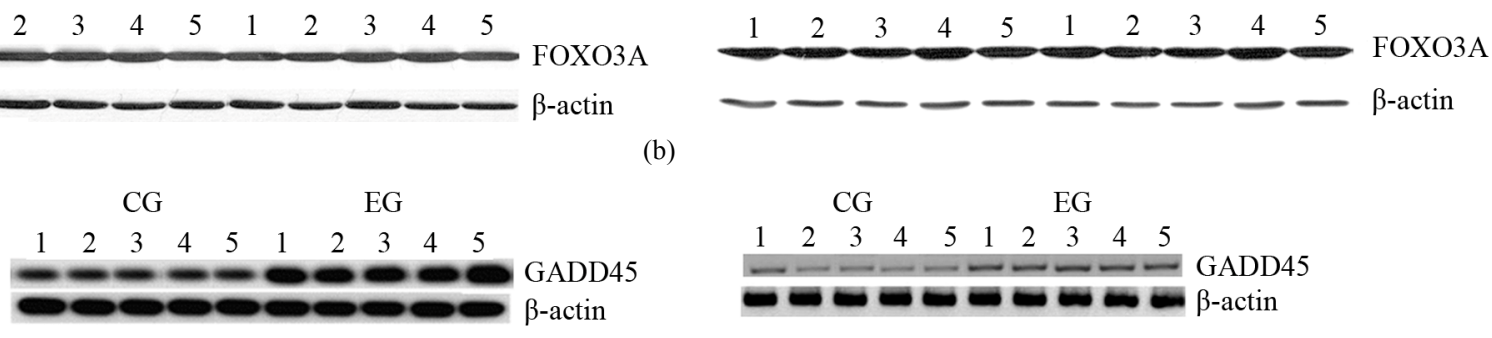

(c)

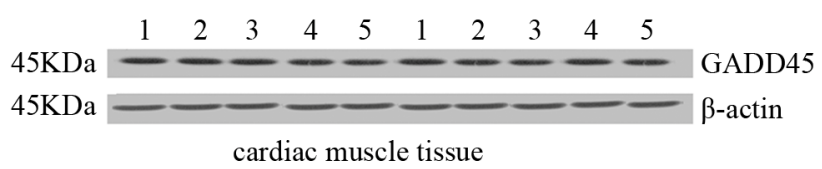

(d)

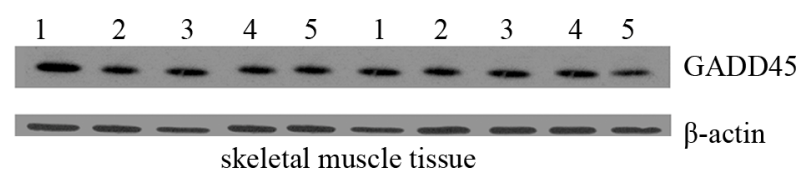

skeletal muscle tissue

Figure 2. Effects of run to exhaustion in rat cardiac and skeletal muscle tissues on the FOXO3A and GADD45 mRNA and protein expression levels. Northern blot and Western blot analysis were performed. $\beta$-actin was used as an internal loading control. (a) Total mRNA was extracted from tissues of CG and EG rats and analyzed Northern blot. The membrane was hybridized with a ${ }^{32} \mathrm{P}$ labeled probe for FOXO3A; (b) Whole cell lysates were prepared from tissues of CG and EG rats and analyzed by Western blot using FOXO3A specific antibodies. $\beta$-actin was used as a control for protein loading. The molecular weight (in KDa) of protein size standards is shown on the left hand side; (c) Total mRNA was extracted from tissues of CG and EG rats and analyzed Northern blot. The membrane was hybridized with a ${ }^{32} \mathrm{P}$ labeled probe for GADD45; (d) Whole cell lysates were prepared from tissues of CG and EG rats and analyzed by Western blot using GADD45 specific antibodies. $\beta$-actin was used as a control for protein loading. The molecular weight (in $\mathrm{KDa}$ ) of protein size standards is shown on the left hand side.

on one side SIRT1 activation may be more important in the cardiac and skeletal muscle adaptations to exercise and on other side could protect PARP-mediated cell death by replenishing cellular NAD levels.

Several evidences, coming from studies on the expression of SIRT1 in failing hearts of human patients and from over-expression studies of SIRT1 in mouse, show that SIRT1 and SIRT7 may act together. For this reason, we examined SIRT7 mRNA and protein expression following prolonged and exhaustive exercise in both cardiac and skeletal muscle tissues. Again, we found that SIRT7 mRNA and protein expression increased after exercise. This result suggests that SIRT7 could have a similar role of SIRT1 after prolonged and exhaustive exercise. Therefore, we can hypothesize that SIRT1 and SIRT7 might play a critical role in adaptations to exercise in both tissues. In addition, an increase in SIRT1 mRNA levels could exert an antioxidant effect. Brunet et al. (2004) [17] demonstrated that in mammalian cells SIRT1 appears to control the cellular response to stress by regulating FOXO transcription factors that function as sensors of the insulin signaling pathway and as regulators of longevity. In particular, these authors showed that SIRT1 and FOXO3A form a complex in response to oxidative stress stimulus. Mammalian FOXO factors control several biological fu- nctions, such as cell cycle arrest, detoxification of ROS and repair of damaged DNA. SIRT1 increased FOXO3A ability to induce cell cycle arrest and enhanced expression of a FOXO3A target gene involved in DNA repair, such as GADD45. Because it is known that exercise exerts its beneficial effects particularly on the cardiovascular system, we tested FOXO3A and its targets involvement. Our data showed a different expression pattern between SIRT1 and FOXO3A. In addition, prolonged and exhaustive exercise did not increase both FOXO3A and GADD45 protein expression in the cardiac and muscle tissues in EG. This finding could be related to higher oxidative stress in samples that would induce to choose apoptosis or necrosis rather than repair as mechanism of detoxification.

Furthermore, it is also established that sirtuins interact with and regulate the activity of FOXO gene family proteins [23]. Since SIRT1 itself does not bind to DNA directly, targeted deacetylation of histones is thought to occur through its interaction with specific DNA binding factors, such as FOXO3A. Thus, since SIRT1 can bind and deacetylate FOXO3A, leading to a selective augmentation of FOXO-regulated stress resistance genes, herein we examined the evolving and relationship between SIRT1 and FOXO3A following the exercise. Our data showed a 


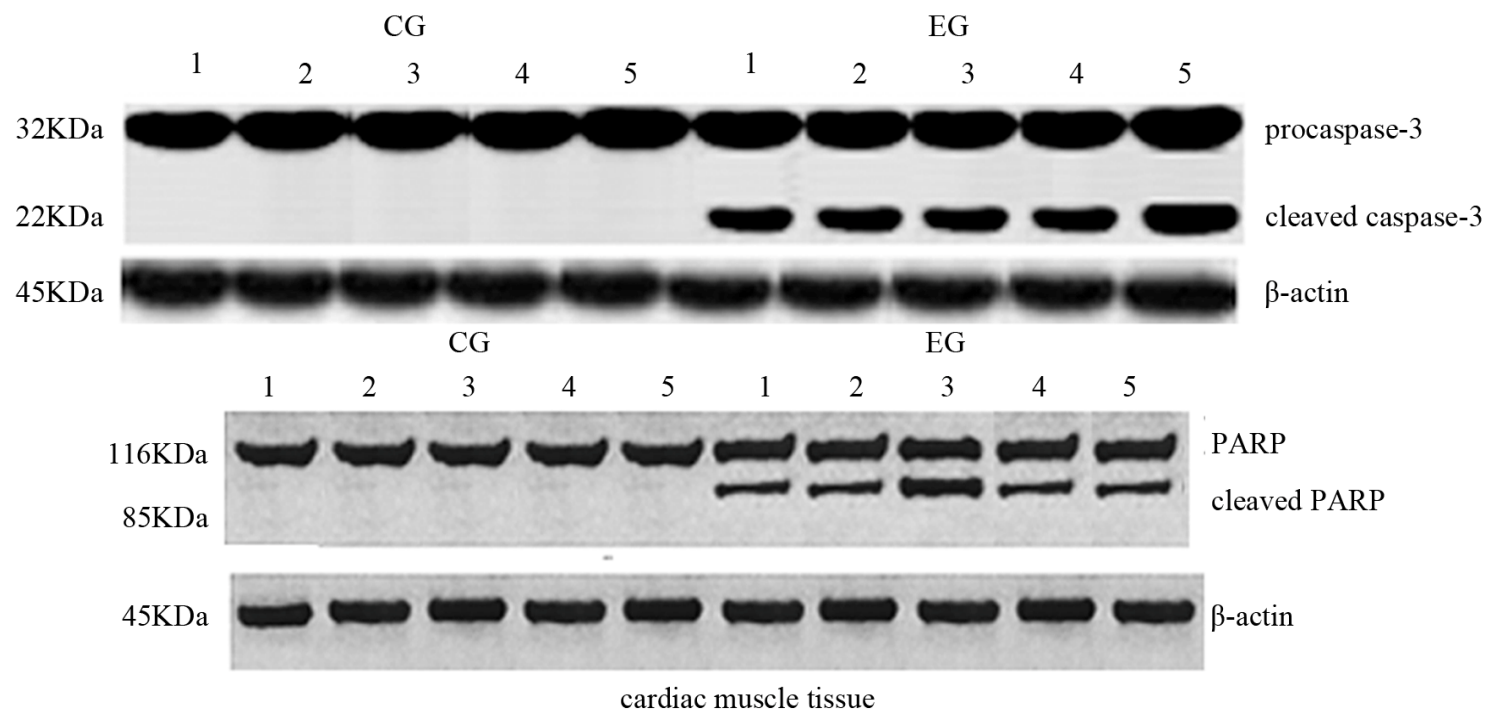

(a)
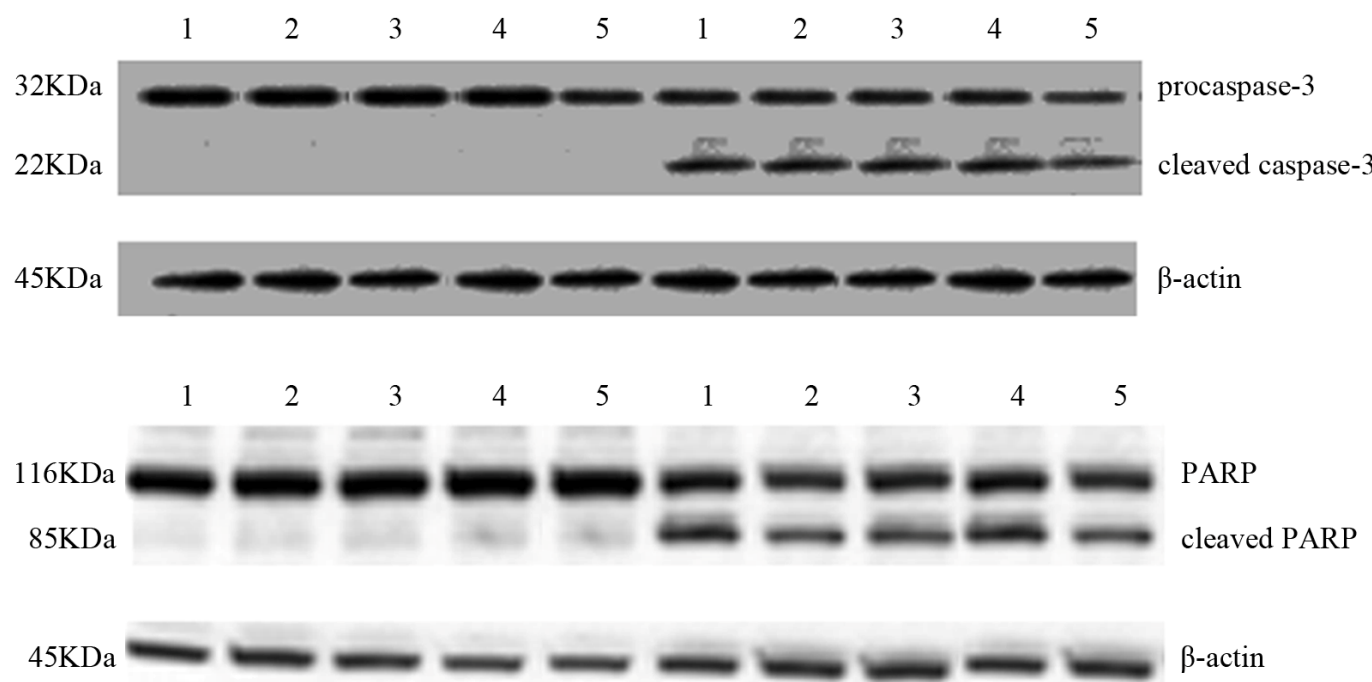

skeletal muscle tissue

(b)

Figure 3. Effects of run to exhaustion in cardiac and skeletal muscle tissues of CG and EG rats on caspase-3 activation and PARP cleavage. Whole cell lysates were prepared from tissues of CG $(a, b)$ and EG $(a, b)$ and analyzed by Western blot using caspase-3 and PARP specific antibodies. $\beta$-actin was used as a control for protein loading. The molecular weight (in $\mathrm{KDa}$ ) of protein size standards is shown on the left hand side.

different expression pattern between SIRT1 and FOXO3A. In fact exercise did not increase FOXO3A protein expression in the cardiac and muscle tissues in EG. Furthermore, it was recently reported that the FOXO3A mediates expression of gene involved in DNA repair such as GADD45. Again, the exercise increased mRNA levels of GADD45 in EG in both tissues but not protein expression levels. Increases of mRNA transcripts do not always correspond to high levels of protein expression because there are multiple points which regulate protein expression following transcription.
Moreover, the time necessary for mRNA to translate and increase functional protein remains unclear and may vary between genes. Since we sampled muscle and cardiac tissues immediately post-exercise this would unlikely give enough time for protein expression to occur equally in both upstream and downstream elements [24].

Another aim of this research was to understand the intense exercise-induced effects on pro-apoptotic and antiapoptotic proteins. We observed caspase-3-activation, cleavage of PARP and an alteration of pro-apoptotic and anti-apoptotic protein expression immediately after the 

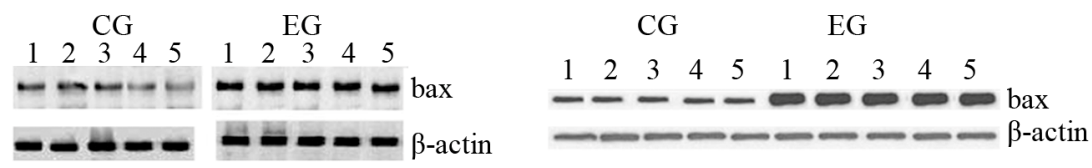

(a)
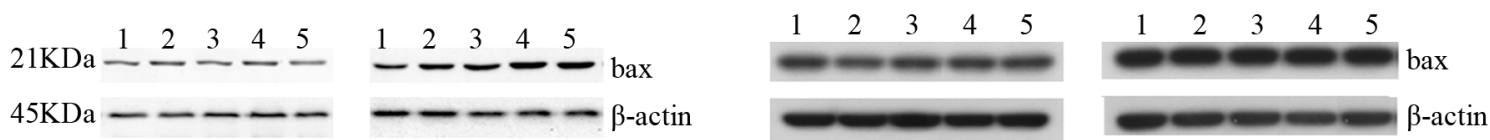

(b)

CG EG
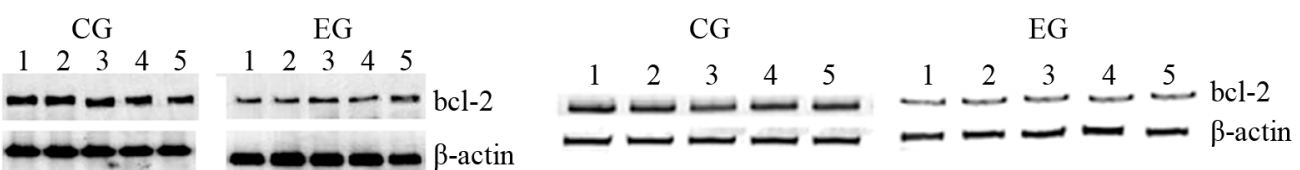

(c)

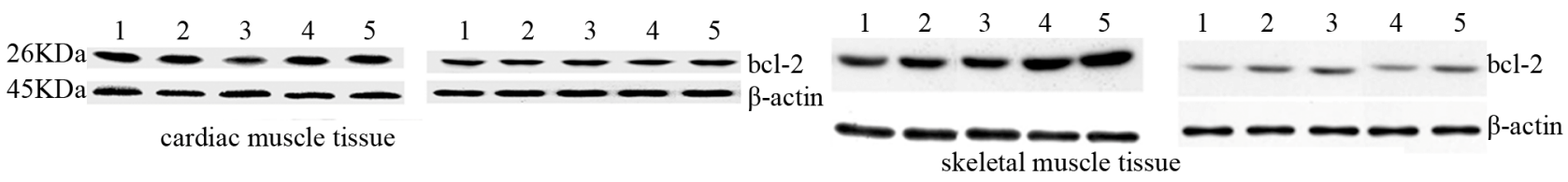

(d)

Figure 4. Effects of run to exhaustion in rat cardiac and skeletal muscle tissues on the bax and bcl-2 mRNA and protein expression levels. Northern blot and Western blot analysis were performed. $\beta$-actin was used as an internal loading control. (a) Total mRNA was extracted from tissues of CG and EG rats and analyzed Northern blot. The membrane was hybridized with a ${ }^{32} \mathrm{P}$ labeled probe for bax; (b) Whole cell lysates were prepared from tissues of CG and EG rats and analyzed by Western blot using bax specific antibodies. $\beta$-actin was used as a control for protein loading. The molecular weight (in KDa) of protein size standards is shown on the left hand side; (c) Total mRNA was extracted from tissues of CG and EG rats and analyzed Northern blot. The membrane was hybridized with $\mathrm{a}^{32} \mathrm{P}$ labeled probe for bcl-2; (d) Whole cell lysates were prepared from tissues of CG and EG rats and analyzed by Western blot using bcl-2 specific antibodies. $\beta$-actin was used as a control for protein loading. The molecular weight (in KDa) of protein size standards is shown on the left hand side.

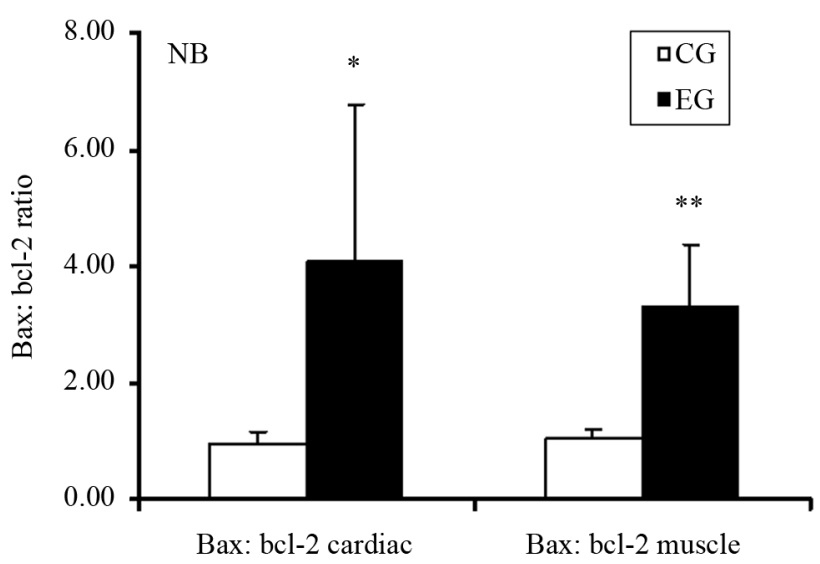

(a)

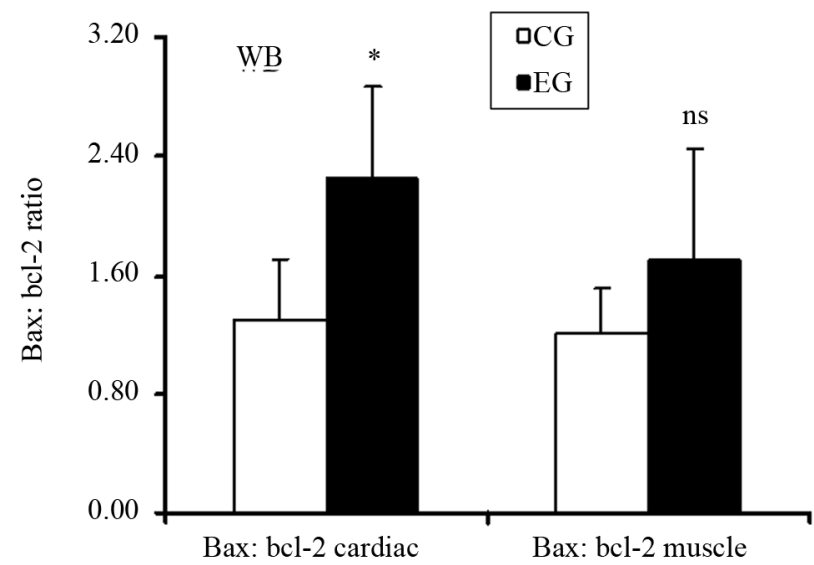

(b)

Figure 5. Effects of run to exhaustion in rat cardiac and skeletal muscle tissues on bax:bcl-2 ratio. (a) Bax:bcl2 ratio in Northern blot analysis in cardiac and skeletal muscle tissue between two groups. ${ }^{* * *} \mathrm{p}<0.001$, different from control and experimental groups; (b) Bax:bcl 2 ratio Western blot analysis in cardiac and skeletal muscle tissue between two groups. ${ }^{* * *} \mathrm{p}<0.001$, different from control and experimental groups.

exercise protocol. As matter of fact, our data showed an increase in bax expression and a slight down-regulation of bcl-2 and thus an increase in their ratio. In conclusion, the constitutive balance ratio of bax:bcl-2 was affected by this kind of exercise. Thus, the bax:bcl-2 ratio can be an important cellular marker that correlates with responsiveness to exercise. Apoptosis has been documented in different tissues after exercise, however it is unclear if cells of different tissues are indeed entirely lost by an apoptotic process or just temporarily "marked" as under- 
going apoptosis. Thus, the increase of SIRT1 and SIRT7 expression level after intense exercise documented herein, could be a protective mechanism started in several tissues to counterbalance the cellular damage or apoptosis induced by various stressor. The results of this study highlight that the apoptosis response to exhaustive stress occurs immediately after the cessation of exercise protocol with increases in caspase-3, PARP and bax and decreases in bcl-2. However, there is an increase of sirtuin proteins, which might have a protective role after an exhaustive exercise. Although the sequence of responses was likely related, further experiments will be needed to demonstrate a causal relationship between the exercise stress, the expression of pro and anti-apoptotic proteins, and the increased expression of sirtuins.

\section{ACKNOWLEDGEMENTS}

The authors would also like to thank Professor José Alberto Duarte for his invaluable contribution with this investigation

\section{REFERENCES}

[1] Gomez-Cabrera, M.C., Viña, J. and Ji, L.L. (2009) Interplay of oxidants and antioxidants during exercise: Implications for muscle health. Physician and Sportsmedicine, 37, 116-123. doi:10.3810/psm.2009.12.1749

[2] Huang, C.C., Lin, T.J., Chen, C.C. and Lin, W.T. (2009) Endurance training accelerates exhaustive exercise-induced mitochondrial DNA deletion and apoptosis of left ventricle myocardium in rats. European Journal of Applied Physiology, 107, 697-706. doi:10.1007/s00421-009-1177-4

[3] Neto, R.J.C., Lira, F.S., Oyama, L.M., Zanchi, N.E., Yamashita, A.S., Batista, M.L. Jr., do Nascimento, C.M.O. and Seelaender, M. (2009) Exhaustive exercise causes an anti-inflammatory effect in skeletal muscle and a pro-inflammatory effect in adipose tissue in rats. European Journal of Applied Physiology, 106, 697-704. doi:10.1007/s00421-009-1070-1

[4] Higashihara, A., Ono, T., Kubota, J., Okuwaki, T. and Fukubayashi, T. (2010) Functional differences in the activity of the hamstring muscles with increasing running speed. Journal of Sports Science and Medicine, 28, 10851092. doi:10.1080/02640414.2010.494308

[5] Kimball, S.R. and Jefferson, L.S. (2010) Control of translation initiation through integration of signals generated by hormones, nutrients, and exercise. Journal of Biological Chemistry, 285, 29027-29032.

[6] Reichhold, S., Neubauer, O., Bulmer, A.C., Knasmüller, S. and Wagner, K.H. (2009) Endurance exercise and DNA stability: Is there a link to duration and intensity? Mutation Research, 682, 28-38. doi:10.1016/j.mrrev.2009.02.002

[7] Marfe, G., Tafani, M., Pucci, B., Di Stefano, C., Indelicato, M., Andreoli, A., Russo, M.A., Sinibaldi-Salimei, P. and Manzi, V. (2010) The effect of marathon on
mRNA expression of anti-apoptotic and pro-apoptotic proteins and sirtuins family in male recreational longdistance runners. BMC Physiology, 10, 7. doi:10.1186/1472-6793-10-7

[8] Zou, J., Yuan, J., Lv, S. and Tu, J. (2010) Effects of exercise on behavior and peripheral blood lymphocyte apoptosis in a rat model of chronic fatigue syndrome. Journal of Huazhong University of Science and Technology, 30, 258-264. doi:10.1007/s11596-010-0225-y

[9] Liang, H., Balas, B., Tantiwong, P., Dube, J., Goodpaster B.H., O’Doherty, R.M., DeFronzo, R.A., Richardson, A., Musi, N. and Ward, W.F. (2009) Whole body overexpression of PGC-1alpha has opposite effects on hepatic and muscle insulin sensitivity. American Journal of Physiology_Endocrinology and Metabolism, 96, E945-E954.

[10] Quadrilatero, J., Bombardier, E., Norris, S.M., Talanian, J.L., Palmer, M.S., Logan, H.M., Tupling, A.R., Heigenhauser, G.J. and Spriet, L.L. (2010) Prolonged moderateintensity aerobic exercise does not alter apoptotic signaling and DNA fragmentation in human skeletal muscle. American Journal of Physiology-Endocrinology and Metabolism, 298, E534-E547.

[11] Brancaccio, P., Lippi, G. and Maffulli, N. (2010) Biochemical markers of muscular damage. Clinical Chemistry and Laboratory Medicine, 48, 757-767. doi:10.1515/cclm.2010.179

[12] Little, J.P., Safdar, A., Cermak, N., Tarnopolsky, M.A. and Gibala, M.J. (2010) Acute endurance exercise increases the nuclear abundance of PGC-1alpha in trained human skeletal muscle. American Journal of Physiology-Regulatory, Integrative and Comparative Physiology, 298, R912-R917.

[13] Jørgensen, S.B., Wojtaszewski, J.F., Viollet, B., Andreelli, F., Birk, J.B., Hellsten, Y., Schjerling, P., Vaulont, S., Neufer, P.D., Richter, E.A. and Pilegaard, H. (2005) Effects of alpha-AMPK knockout on exercise-induced gene activation in mouse skeletal muscle. FASEB Journal, 19, 1146-1148.

[14] Tran, H., Brunet, A., Grenier, J.M., Datta, S.R., Fornace, A.J. Jr., DiStefano, P.S., Chiang, L.W. and Greenberg, M.E. (2002) DNA repair pathway stimulated by the forkhead transcription factor FOXO3A through the GADD45 protein. Science, 296, 530-534. doi:10.1126/science.1068712

[15] Lappalainen, Z. (2011) Sirtuins: A family of proteins with implications for human performance and exercise physiology. Research in Sports Medicine, 19, 53-65. doi:10.1080/15438627.2011.536068

[16] Freyssenet, D. (2007) Energy sensing and regulation of gene expression in skeletal muscle. Journal of Applied Physiology, 102, 529-540. doi:10.1152/japplphysiol.01126.2005

[17] Brunet, A., Sweeney, L.B., Sturgill, J.F., Chua, K.F., Greer, P.L., Lin, Y., Tran, H., Ross, S.E., Mostoslavsky, R., Cohen, H.Y., Hu, L.S., Cheng, H.L., Jedrychowski, M.P., Gygi, S.P., Sinclair, D.A., Alt, F.W. and Greenberg, M.E. (2004). Stress-dependent regulation of FOXO transcription factors by the SIRT1 deacetylase. Science, 303, 2011-2015. doi:10.1126/science.1094637 
[18] Lee, J.H., Song, M.Y., Song, E.K., Kim, E.K., Moon, W.S., Han, M.K., Park, J.W., Kwon, K.B. and Park, B.H. (2009) Overexpression of SIRT1 protects pancreatic betacells against cytokine toxicity by suppressing the nuclear factor-kappaB signaling pathway. Diabetes, 58, 344-351. doi:10.2337/db07-1795

[19] Radak, Z., Chung, H.Y. and Goto, S. (2008) Systemic adaptation to oxidative challenge induced by regular exercise. Free Radical Biology and Medicine, 44, 153-159. doi:10.1016/j.freeradbiomed.2007.01.029

[20] Suwa, M., Nakano, H., Radak, Z. and Kumagai, S. (2008) Endurance exercise increases the SIRT1 and peroxisome proliferator-activated receptor gamma coactivator-1alpha protein expressions in rat skeletal muscle. Metabolism, 57, 986-998. doi:10.1016/j.metabol.2008.02.017

[21] Vakhrusheva, O., Smolka, C., Gajawada, P., Kostin, S., Boettger, T., Kubin, T., Braun, T. and Bober, E. (2008) Inflammatory cardiomyopathy in mice SIRT7 increases stress resistance of cardiomyocytes and prevents apop- tosis and inflammatory cardiomyopathy in mice. Circulation Research, 102, 703-710.

[22] Pillai, J.B., Gupta, M., Rajamohan, S.B., Lang, R., Raman, J. and Gupta, M.P. (2006) Poly(ADP-ribose) polymerase-1-deficient mice are protected from angiotensin II-induced cardiac hypertrophy. American Journal of Physiology - Heart and Circulatory Physiology, 291, H1545H1553.

[23] Wang, F., Nguyen, M., Qin, F.X. and Tong, Q. (2007) SIRT2 deacetylates FOXO3A in response to oxidative stress and caloric restriction. Aging Cell, 6, 505-514. doi:10.1111/j.1474-9726.2007.00304.x

[24] Dumke, C.L., Mark, D.J., Angela, M.E., Nieman, D.C., Carmichael, M.D., Quindry, J.C., Travis, T.N., Utter, A..C, Gross, G.S.J., Henson, D.A., McAnulty, S.R. and McAnulty, L.S. (2009) Successive bouts of cycling stimulates genes associated with mitochondrial biogenesis. European Journal of Applied Physiology, 107, 419-427. doi:10.1007/s00421-009-1143-1

\section{Supplementary Material}

\section{MATERIAL AND METHODS}

\subsection{Tissue Collection}

Rats were anesthetized with sodium thiopental $(60 \mathrm{mg} / \mathrm{kg}$, i.p.) and placed in a supine position. After a quick opening chest, the whole heart was rapidly excised, rinsed with ice-cold saline, carefully dried. Then, the atria and the great vessels were removed and the ventricles stored at $-80^{\circ} \mathrm{C}$ for posterior biochemical analysis. From each animal, both intact gastrocnemius muscles were also collected for further biochemical assays. Then, the tissues were minced and homogenized with an Ultra-Turrax homogenizer.

\subsection{RNA Isolation and Northern Blot Analysis}

Total RNAs from samples were isolated using Tri Reagent (Sigma-Aldrich Chemie GmbH, Taufkirchen, Germany). Aliquots of RNA were electrophoresed on 1\% agarose formaldehyde gels and subsequently blotted onto nylon membranes (Hybond N, Amersham, Braunschweig, Germany). The membrane was then UV cross-linked, and hybridized to ${ }^{32} \mathrm{P}$-labelled probe. The relative amount of protein expression was quantified using a Gel-Doc phosphorimager and Quantity One software (Bio-Rad) and normalized by the intensity of $\beta$-actin.

\subsection{Protein Extraction and Western Blot Analysis}

Tissues were homogenized directly into lysis buffer (50 $\mathrm{mM}$ HEPES, $150 \mathrm{mM} \mathrm{NaCl}, 1 \mathrm{mM}$ EDTA, $1 \mathrm{mM}$ EGTA, 10\% glycerol, 1\% NP-40, $1 \mathrm{mM}$ phenylmethylsulfonyl fluoride, $1 \mu \mathrm{g} / \mathrm{ml}$ aprotinin, $0.5 \mathrm{mM}$ sodium orthovanadate, and $20 \mathrm{mM}$ sodium pyrophosphate). The lysates were centrifugated at $14000 \times \mathrm{rpm}$ for $10 \mathrm{~min}$. Protein concentrations were determined by the Bradford assay (Bio-Rad, Hercules, CA). Equivalent amounts of proteins were loaded and electrophoresed on SDS-polyacrylamide gels. Subsequently, proteins were transferred to nitrocellulose membranes (Immobilon, Millipore Corp., Bedford, MA). After blocking with Tris-buffered salineBSA (25 mM Tris (pH 7.4), $200 \mathrm{mM} \mathrm{NaCl}$, and 5\% BSA), the membrane was incubated with the following primary antibodies: Anti-Lactate Dehydrogenase (LDH) PAb (dilution 1:1500) and anti-b-actin MAb (1:7500) (ABCAM Cambridge UK). Membranes were then incubated with the appropriate horseradish peroxidase-conjugated secondary antibody (dilution 1:1000) (at room temperature), and the reaction was detected with an enhanced chemiluminescence system (Amersham Life Science, Buckinghamshire, UK). The relative amount of protein expression was quantified using a Gel-Doc phosphorimager and Quantity One software (BIO-RAD Philadelphia USA) and normalized by the intensity of $\beta$-actin. 

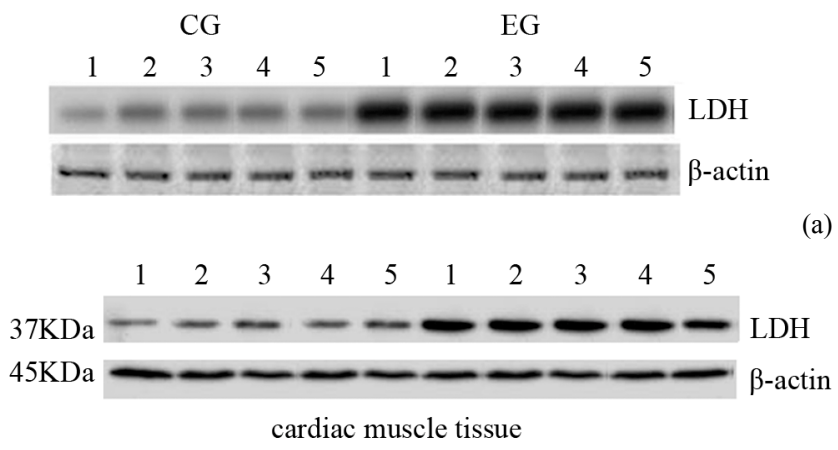

(a)
CG EG
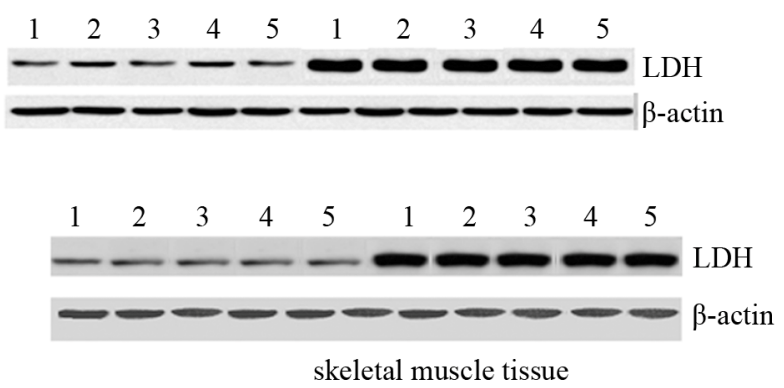

(b)

Figure 1S. Effects of run to exhaustion in rat cardiac and skeletal muscle tissues on the LDH mRNA and protein expression levels. Northern blot and Western blot analysis were performed. $\beta$-actin was used as an internal loading control. (a) Total mRNA was extracted from tissues of CG and EG rats and analyzed Northern blot. The membrane was hybridized with a ${ }^{32} \mathrm{P}$ labeled probe for LDH; (b) Whole cell lysates were prepared from tissues of CG and EG rats and analyzed by Western blot using LDH specific antibodies. $\beta$-actin was used as a control for protein loading. The molecular weight (in KDa) of protein size standards is shown on the left hand side.

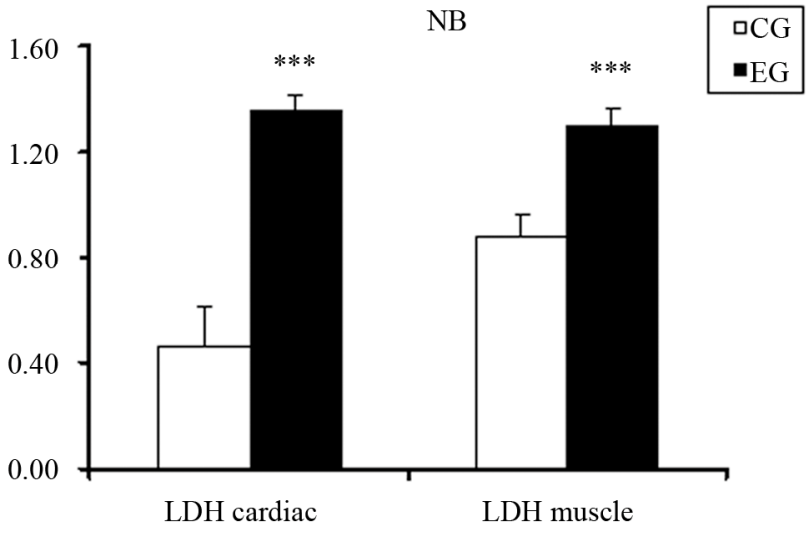

(a)

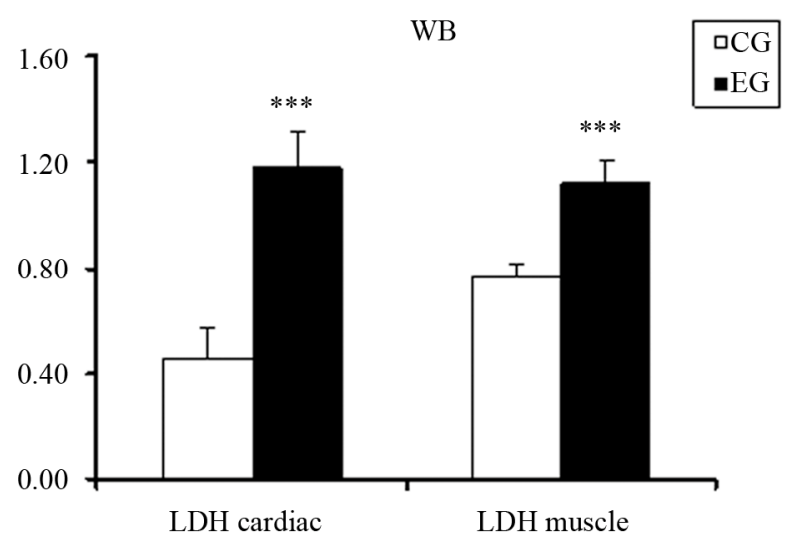

(b)

Figure 2S. Effects of run to exhaustion in rat cardiac and skeletal muscle tissues on LDH mRNA transcript and expression levels. (a) Northern Blot analysis of LDH in cardiac and skeletal muscle tissue between two groups. ${ }^{* * *} \mathrm{p}<0.001$, different from control and experimental groups; (b) Western Blot analysis of LDH in cardiac and skeletal muscle tissue between two groups. ${ }^{* * *} \mathrm{p}<0.001$, different from control and experimental groups between $\mathrm{CG}$ and $\mathrm{EG}$. 